\title{
Nanoparticle-functionalized dressings for the treatment of third-degree skin burns - histopathological and immunohistochemical study
}

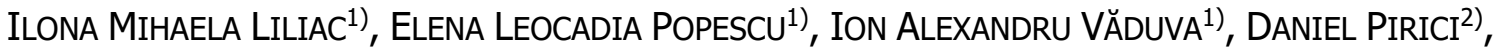

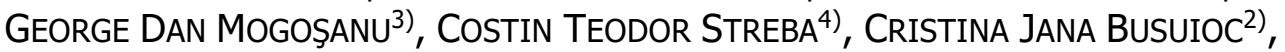

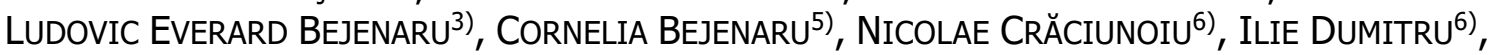 \\ HAZIM ELAYAN ${ }^{7)}$, LAURENTIU MOGOANTĂ ${ }^{2)}$
}

\author{
1) PhD Student, Doctoral School, University of Medicine and Pharmacy of Craiova, Romania \\ 2) Department of Histology, University of Medicine and Pharmacy of Craiova, Romania \\ ${ }^{3}$ Department of Pharmacognosy \& Phytotherapy, Faculty of Pharmacy, University of Medicine and Pharmacy of Craiova, \\ Romania \\ 4) Department of Pulmonology, University of Medicine and Pharmacy of Craiova, Romania \\ 5) Department of Pharmaceutical Botany, Faculty of Pharmacy, University of Medicine and Pharmacy of Craiova, Romania \\ 6) Department of Road Vehicles, Transportation and Industrial Engineering, Faculty of Mechanics, University of Craiova, \\ Romania \\ 7)Department of Plastic Surgery, ArcaLife Plastic Surgery Clinic, Bucharest, Romania
}

\begin{abstract}
Skin burns are one of the most common injuries associated with increased morbidity and mortality, especially in the children and the elderlies. Severe burns, especially, result in a systemic immune and inflammatory response, which may reflect in multiple organ insufficiency, and a fast and effective local restorative process is essential for functionality recovering, as well as for interrupting the generalized systemic response. We have aimed here to assess the effect of different wound dressings in what it regards the morphology and clinical restoration after a skin burn. On a rat animal model, we have evaluated the macroscopic and histopathological features of controlled third degree skin burns in control animals versus treatments with local dressings of silver sulfadiazine (SDA) cream, simple gel (G), gel + silver nanoparticles (AgNPs) (G+NPS), gel + exosomes $(\mathrm{G}+\mathrm{EXO})$ and gel + AgNPs + exosomes (Gel+NPS+EXO), at 14 days and, respectively, 21 days after the lesion. Tissue fragments were harvested and processed for histopathology and immunohistochemistry. Immunofluorescence was utilized to evaluate the maturity of underlaying granulation tissue based on double stainings for smooth muscle actin (SMA) and cluster of differentiation 31 (CD31). Our study showed variability in what it regards the vessel density and immunoexpression of SMA between the treatments, and image analysis revealed that most SMA reduction and blood vessel density reduction in the maturing granulation tissue occurred for the G+NPS and G+NPS+EXO treatments. A complete re-epithelization was also observed for the G+NPS+EXO treatment. Overall, our results show that improved topic treatments promote faster re-epithelization and reparation of the dermis after skin burn lesions, providing thus an avenue for new treatments that aim both local recuperation and systemic infection prevention.
\end{abstract}

Keywords: burns, skin regeneration, silver nanoparticles, exosomes, animal model.

\section{ㅁ Introduction}

The skin plays a major role in protecting the human and animal organisms against physical, chemical and biological agents aggression from the external environment, as well as in maintaining internal homeostasis, being constantly involved in a wide range of physiological processes, such as hydro-mineral balance, maintaining and regulating temperature, receiving external stimuli from the environment, hormone production and activation, synthesis of vitamin D, neuropeptide and cytokine production, etc. [1-3].

Burns are one of the most common injuries worldwide; it is estimated that over one million individuals in the United States are affected by this disease each year [4]. According to recent data published by the World Health Organization (WHO), skin burns cause between 180000 and 265000 deaths annually, rendering them a global public health problem $[5,6]$.

The annual hospitalization rate for skin burns in Europe is between 2-29 per 1000 persons. The economic impact is significant because burns are difficult to heal, require long periods of hospitalization and involve expensive material costs [5].

Skin burns can result in serious complications, such as sepsis [7-10], hypovolemic shock, acute renal failure, liver or heart failure, retraction of skin tissues after inappropriate wound healing and the appearance of keloid scars, depending on the injured skin area and depth. Lesions of the epidermis lead to a significant loss of body fluids, which contributes to dehydration, electrolyte imbalance and renal and circulatory failure. Furthermore, because to

This is an open-access article distributed under the terms of a Creative Commons Attribution-NonCommercial-ShareAlike 4.0 International Public License, which permits unrestricted use, adaptation, distribution and reproduction in any medium, non-commercially, provided the new creations are licensed under identical terms as the original work and the original work is properly cited. 
the breakdown of physiological protection provided by the layers of the epidermis, burnt skin is especially vulnerable to bacteria and other pathogens. Each of these complications has the potential to be lethal or to worsen the patient's suffering.

Burns have a substantial negative effect on patients, both physically (due to keloid scars or disabilities) and emotionally [11].

Frequently, large and deep burns lead to the death of the affected person. Thus, in patients with more than $40 \%$ burned body surface, death occurs due to bacterial infection $[12,13]$. Burn patient survival rates have increased significantly in recent decades, due to intensive support of vital organ functions (lungs, kidneys, liver, and heart), through intensive care techniques, as well as the use of various skin grafts that facilitate a quicker recovery of the skin surface. Despite their widespread usage, autologous skin grafts are inadequate for treating severe, extensive burns due to the limited area of skin collecting sites [5].

Recent years have shown the development of innovative therapeutic approaches that integrate nanotechnologies into healthcare, to the point that therapeutic alternatives now include the use of nanoparticles for the treatment of burn infections and the successful healing of skin wounds following exposure [14].

\section{Aim}

The purpose of this study was to characterize the histological and immunohistochemical (IHC) changes that occurred during the skin's healing process after third degree burns in an experimental animal model treated with nanostructured functional dressings.

\section{Materials and Methods}

The study was conducted on laboratory animals, specifically Wistar common rats, adults weighing between $350 \mathrm{~g}$ and $410 \mathrm{~g}$, which were provided by the Animal Facility of the University of Medicine and Pharmacy of Craiova, Romania. The experiments were approved by the Ethics Committee of the University of Medicine and Pharmacy of Craiova. The experimental protocol was applied in accordance with European Council Directive No. 86/609/1986 and the European Convention for the Protection of Vertebrate Animals Used for Experimental and Other Scientific Purposes (December 2, 2005), and the Romanian Parliament-approved Law No. 43/2014 on the Protection of Animals Used for Scientific Purposes. In this regard, it was considered to limit as much as possible the number of animals in the group, performing lesions under general anesthesia and also proper care of post-experimental animals. Throughout the experiment, the animals were monitored in the Animal Facility of the University of Medicine and Pharmacy of Craiova, under standard temperature, humidity, lighting, feeding, and water conditions (ad libitum).

A third degree burn with a diameter of about $1 \mathrm{~cm}$ was performed in 48 rats after hair removal from the dorsal region and after giving them general anesthesia by intramuscular injection with a mixture of Ketamine hydrochloride
$85 \mathrm{mg} / \mathrm{kg}$ body-weight (b.w.) (Ketamidor ${ }^{\circledR} 100 \mathrm{mg} / \mathrm{mL}$, Richter Pharma AG) and Xylazine hydrochloride $6 \mathrm{mg} / \mathrm{kg}$ b.w. (Xylazin Bio ${ }^{\circledR} 2 \%$, Bioveta), using a special device, the electronically controlled heating station, type QUICK $303 \mathrm{D}$, with a temperature range of $50^{\circ} \mathrm{C}$ to $500^{\circ} \mathrm{C}$. The burn was achieved by placing the station's adapter head on the animal's depilated skin for five seconds, while it was heated to $100^{\circ} \mathrm{C}$.

The animals were then divided into two equal batches of 24 animals each, which were monitored for 14 and 21 days after the burn was produced. The groups were randomly divided into six subgroups, each of four animals, as follows:

- group 1.1 - control group, untreated, monitored for 14 days;

- group 2.1 - control group, untreated, monitored for 21 days;

- group 1.2 - treated with silver sulfadiazine (SDA) cream (Dermazin ${ }^{\circledR}$, Sandoz), monitored for 14 days;

- group 2.2 - treated with silver sulfadiazine (SDA) cream (Dermazin ${ }^{\circledR}$, Sandoz), monitored for 21 days;

- group 1.3 - treated with simple gel $(\mathrm{G})$, monitored for 14 days;

- group 2.3 - treated with simple gel $(\mathrm{G})$, monitored for 21 days;

- group 1.4 - treated with gel + silver nanoparticles (AgNPs) (G+NPS), monitored for 14 days;

- group 2.4 - treated with gel + AgNPs (G+NPS), monitored for 21 days;

- group 1.5 - treated with gel + exosomes $(\mathrm{G}+\mathrm{EXO})$, monitored for 14 days;

- group 2.5 - treated with gel + exosomes $(\mathrm{G}+\mathrm{EXO})$, monitored for 21 days;

- group 1.6 - treated with gel + AgNPs + exosomes $(\mathrm{G}+\mathrm{NPS}+\mathrm{EXO})$, monitored for 14 days;

- group 2.6 - treated with gel + AgNPs + exosomes $(\mathrm{G}+\mathrm{NPS}+\mathrm{EXO})$, monitored for 21 days.

Daily treatment was administered to the animals at the level of the burned wound, to observe the macroscopic evolution of the healing process. Both for the control group and for the five local treatment groups, the burned wounds were covered daily with a collagen-based dressing (Hydrocoll ${ }^{\circledR}$, Paul Hartmann AG).

The burnt skin fragments were removed for microscopic and IHC analysis at the completion of the experiment period, respectively 14 and 21 days, and the remaining losses of tissue were surgically sutured under general anesthesia with a mixture of Ketamine and Xylazine. The biological material was fixed for 72 hours in $10 \%$ neutral buffered formalin before being embedded in paraffin, according to the classical histopathology protocol. Subsequently, $4 \mu \mathrm{m}$-thick sections were cut on the Microm HM350 rotary microtome, equipped with a section transfer system on a water bath (Section Transfer System, STS). The slides were stained with Hematoxylin-Eosin (HE) and trichrome based on light green, Goldner-Szekely (GS) technique.

Further sections were prepared for double fluorescence immunohistochemistry to visualize simultaneously the vascular endothelia and smooth muscle actin (SMA) in smooth muscle cells, pericytes and myofibroblasts. After 
rehydration and microwaving in citrate buffer, $\mathrm{pH} 6$, for antigen retrieval, sections were first blocked for 30 minutes in a $1 \%$ hydrogen peroxide solution to quench the endogenous peroxidase. Next, unspecific antibody binding sites were blocked for further 30 minutes in 3\% skimmed milk (BioRad, München, Germany) and then were incubated for 18 hours, at $4^{\circ} \mathrm{C}$, with a mix of primary antibodies [mouse antiSMA, diluted as 1:50, Dako, Glostrup, Denmark, product code M0851; and rabbit anti-cluster of differentiation (CD)31, diluted as 1:100, Novus Antibodies, product code NB100-2284]. The next day, the signal was amplified for two hours using a mix of goat anti-mouse Alexa Fluor 488 (Invitrogen, diluted as 1:150) and goat-anti-rabbit peroxidase labeled polymer (Vector). The peroxidase was visualized with an Alexa 596 conjugated Tyramide amplification step (1:200, five minutes), and the slides were coversliped utilizing a 4',6-Diamidino-2-phenylindole (DAPI)-based antifading medium (Vector).

The sections were imaged with an Eclipse 90i motorized microscope (Nikon, Elta90, Romania) equipped with a 16megapixel color camera (Nikon DS-Ri2), and narrowband fluorescent filters centered for Alexa 594, Alexa 488 and DAPI excitation and emission wavelengths.

The Nikon NIS-Elements Advanced Research package was utilized to control the microscope and camera, and to capture and store the images in the Nikon proprietary format. For analysis, $40 \times$-objective fluorescence images were captured from the granulation tissue areas, taking four images for each animal. For each image, we have next measured the number and diameters of all the vessels that posed a discernable lumen, as well as the area of the SMA signal. All values were averaged for each animal, then for each animal group, and represented graphically as averages \pm standard deviation (SD). All the data were represented graphically utilizing Microsoft Excel 2009 and analyzed utilizing a one-way analysis of variance (ANOVA) followed by Fisher's least significant difference (LSD) test in the Statistical Package for the Social Sciences (SPSS) software. In all cases, $p<0.05$ was used to indicate statistical significance.

\section{ㅁ Results}

All animals were clinically monitored by assessing the healing process of the burn wound. No animals died during the experiment. Macroscopically, the animals treated with G+NPS and G+NPS+EXO had a favorable wound evolution, with the wound completely healed after 21 days, whereas the animals treated with G+EXO also had a favorable wound evolution, with the wound nearly completely healed after 21 days.

The control group, the group treated with simple gel $(\mathrm{G})$, and the reference group treated with SDA cream, all demonstrated a delay in wound healing, with incomplete epithelialization at 21 days. After three weeks of topical administration, we consider that the beneficial development of burn wounds in experimental groups G+NPS and $\mathrm{G}+\mathrm{NPS}+\mathrm{EXO}$ is mostly attributable to the presence of AgNPs, which have antibacterial and wound healing properties (granulation process).

Microscopic examination of histopathological slides on standard (HE) staining confirmed the favorable evolution of burn wounds, as evidenced by the presence of abundant granulation tissue in different stages of maturation occupying the entire thickness of burnt skin, from depth to surface, in batches harvested at 14 and 21 days after injury. In the region of the burned area, the complete loss of skin appendages (sebaceous glands, sweating, hair follicle) was noticed in all groups of animals.

The granulation tissue consisted of a young connective tissue, rich in intercellular conjunctival matrix, with numerous fibroblasts, immune system cells (especially lymphocytes, plasma cells, macrophages, but also rare neutrophilic granulocytes), numerous angiogenesis-related young blood vessels, and connective fibers, predominantly collagen fibers. The appearance of the granulation tissue varied between the animal groups, and even from one animal to another, aspects that may be due to the particular reactivity of each animal to thermal aggression. Also, there were clear differences between the animals in the 14-day group and the animals in which the burned skin was harvested at 21 days.

Although it is recognized that in the case of skin burns with a metallic heat source, the largest lesions occur at the edge of the burn, where caloric energy tends to discharge more intensely, in our study we noticed that the most intense lesions occurred in the center of the burn and the wound healing process developed from the depth of the wound to the surface and from the edges of the wound to its center.

The burn wound healing process involved the production and maturation of granulation tissue (which filled in the injured connective tissues of the dermis and hypodermis like a lead), as well as the appearance and maturation of the epidermis. It could be observed that (Figure 1, A-F), at 14 days, in the control group (Figure 1A) and the SDAtreated group (Figure 1B), there was a young granulation tissue, rich in inflammatory cells and an early epithelialization process. In the simple gel-treated group, a thin epithelium developed beneath the overlaying burned crust, partially detached from the granulation tissue (Figure 1C), indicating the presence of an immature and unstructured basement membrane. The granulation tissue appeared more mature in the groups treated with $\mathrm{G}+\mathrm{NPS}, \mathrm{G}+\mathrm{EXO}$, or $\mathrm{G}+\mathrm{NPS}+\mathrm{EXO}$, the basement membrane appeared more structured, the inflammatory type cells were numerically low, and the epidermis appeared more structured (Figure 1, D-F).

Histopathological examination of skin burns after 21 days revealed that the epidermis regenerated in all groups of animals, but its stratification was reduced in the control group, SDA-treated group, and simple gel-treated group. Additionally, in these cases, the granulation tissue in dermis was immature, revealing many blood vessels or being invaded by the inflammatory cells (Figures 2, A-C).

On the other hand, in the groups treated with $\mathrm{G}+\mathrm{EXO}$, G+NPS or G+NPS+EXO, a complete epithelialization was noticed with the highlighting of the granular and corneous layer of the epidermis. In the last group, treated with $\mathrm{G}+\mathrm{NPS}+\mathrm{EXO}$, the appearance of dermal papillae and an incipient process of regeneration of the epidermal appendages, 
respectively of the pilosebaceous follicles were noticed (Figures 2, D-F).

We have next evaluated blood vessels features by double immunofluorescence for SMA and CD31 at both 14 days (Figure 3, A-F) and 21 days (Figure 4, A-F) postlesion times. At 14 days, there were global statistically significant differences between vessel diameter in the granulation tissue under the lesion, $\mathrm{F}(5,17)=4.139, p=0.02$ (Figure 5A). Treatments with simple gel, G+EXO and SDA had the highest average vessel diameters, homogenous and without statistically significant differences between these groups. SDA and G+EXO had significantly higher diameters than control tissue $(p<0.05)$. G+NPS, on the other hand, showed the smallest values in the group, and the difference attained statistical significance when compared between SDA, simple gel, and $\mathrm{G}+\mathrm{EXO}$ groups $(p<0.05)$.

When counting the number of vessels, the G+EXO animals tended to have the highest vascular densities (21.33/ $\pm 0.58 / 40 \times$ area), and the G+NPS the smaller densities $(6.33 / \pm 1.53 / 40 \times$ area $)$, however, due to the high variability, there were no statistically significant differences between these groups for the number of vessels ( $p>0.05)$ (Figure 5B).

There were statistically significant differences between the SMA areas in the granulation tissue under the lesion, $\mathrm{F}(5,17)=57.305, p<0.001$ (Figure $5 \mathrm{C}$ ). $\mathrm{G}+\mathrm{EXO}$ was showing significantly more SMA values than all the other groups, $p<0.001$. SDA group showed the second highest value, with significant differences from control, G+EXO, G+NPS and $\mathrm{G}+\mathrm{NPS}+\mathrm{EXO}$ groups $(p<0.05)$. Control groups showed the smallest SMA value, with significant differences compared to SDA, simple gel and G+EXO groups $(p<0.05)$.
At 21 days, vessel diameter measurements showed that simple gel groups had the tendency for the largest vessels recorded, while G+NPS group showed the smallest values, however, due to the heterogeneity, there were no global significant differences $[\mathrm{F}(5,17)=3.303, p=0.051]$ (Figure 5D). As opposite from the measurements at 14 days, at 21 days the number of vessels were clearly different between different animal groups $[\mathrm{F}(5,17)=13.751, p<0.001]$ (Figure 5E). The control group had the highest number of vessels, all the treatments seemed to decrease to different extents the vascular density, and only SDA and G+NPS+EXO showed non-significant differences compared to control animals $(p>0.05)$. On the other hand, G+EXO and G+NPS showed the smallest values in the group $(p<0.05)$, however when combining EXO with NPS showed a robust increase of the vessel density $(p<0.05)$. SMA total expression area showed significant global differences between groups, although $\mathrm{G}+\mathrm{EXO}$ group had a large variation of signal $[\mathrm{F}(5,17)=4.432$, $p=0.26]$ (Figure 5F). Simple gel and G+NPS+EXO groups showed close related expression values, while SDA and G+NPS groups had the smallest expression values in the series.

Altogether, at 21 days, if we compare the treatments that showed the most SMA reduction and also combined a maturation of the granulation tissue (i.e., blood vessel density reduction), G+NPS seems to be most effective treatment, followed by G+NPS+EXO model. It is important to observe here that also at 14 days these two treatments showed the smallest SMA values (Figure 5C), however, the G+NPS also showed a premature blood vessel density decrease (Figure 5B).

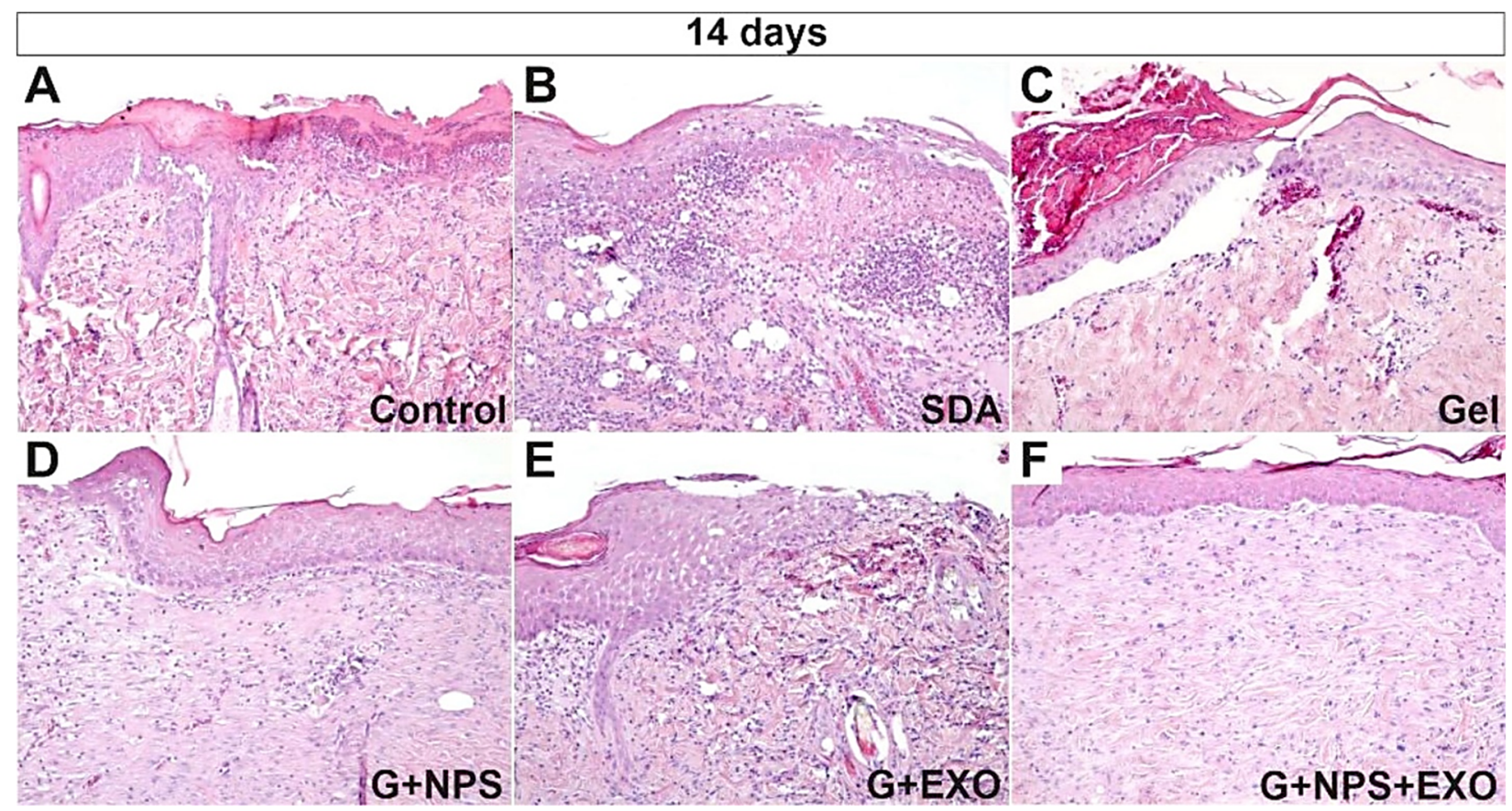

Figure 1 - Histopathology of the skin at 14 days after the lesion. There was mostly a necrotic epidermis, with maturing granulation tissue, with numerous immune cells infiltrating the dermis for control animals (A), with more abundant epithelial necrosis, and liquefaction of the dermis with follicular-like inflammatory infiltrate in SDA group (B), with higher density collagen for simple gel group $(C)$, well-preserved epidermis and scant granulation tissue vessels for G+NPS group (D), thick epidermis and densely packed bundles of collagen fibers for the G+EXO group (E), and a continuous epidermis with well-organized collagen fiber bundles in the dermis for G+NPS+EXO group $(F)$. HE staining: $(A-F) \times 100$. EXO: Exosomes; G: Gel; HE: Hematoxylin-Eosin; NPS: Silver nanoparticles; SDA: Silver sulfadiazine. 


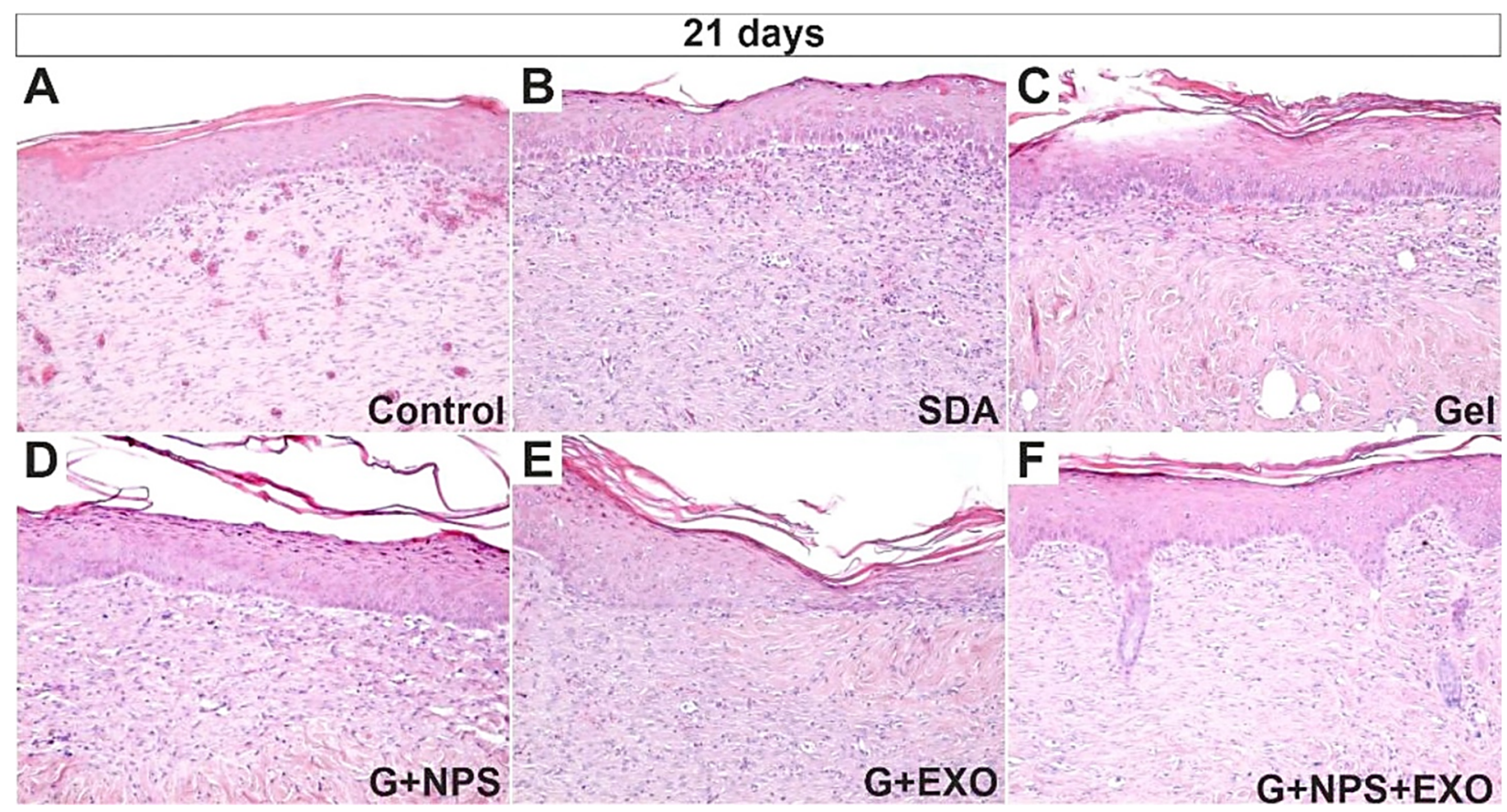

Figure 2 - Histopathology of the skin at 21 days after the lesion showed complete epithelial restauration for all groups. Stasis and scant inflammatory infiltrate can be identified in the control group (A), still immature granulation tissue in the underlying dermis for SDA group (B), scant inflammatory infiltrate in the upper dermis and mostly highly organized collagen bundles in the deeper dermis for the simple gel group $(C)$, partially mature granulation tissue with minimal collagen bundle formation for the G+NPS group (D), regulate but dense collagen fiber bundles in the superficial dermis of the $G+E X O$ group $(E)$, and complete epidermis-dermis regeneration with the apparition of dermic papillae and epithelial crests for the G+NPS+EXO group (F). HE staining: $(A-F) \times 100$. EXO: Exosomes; G: Gel; HE: Hematoxylin-Eosin; NPS: Silver nanoparticles; SDA: Silver sulfadiazine.

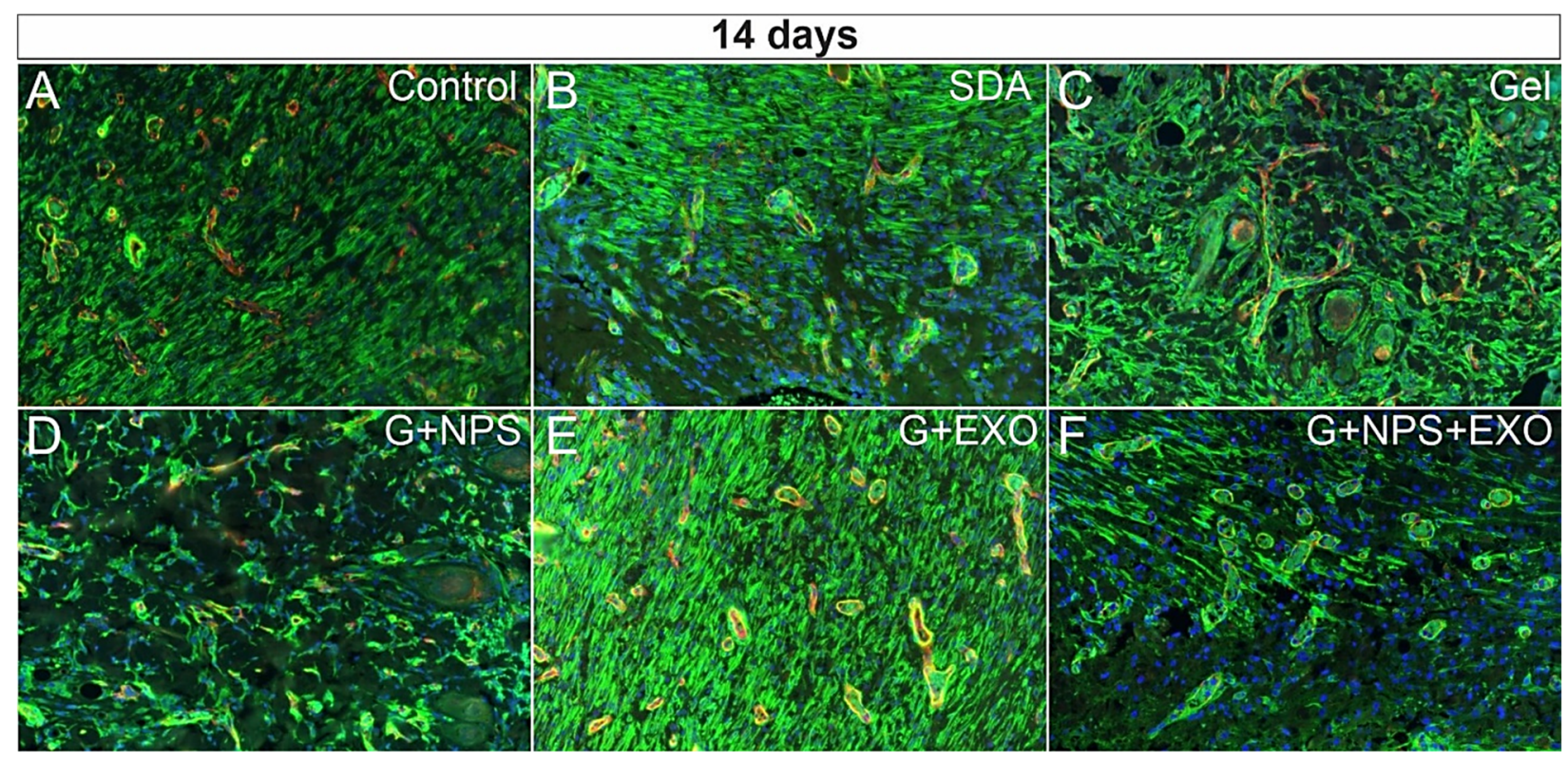

Figure 3 - SMA (green) and CD31 (red) co-expression by fluorescence immunohistochemistry $(\times 200)$ in control group (A) and the five treatment groups $(B-F)$ at 14 days after the lesion. An increase in the SMA immunoexpression was visible for most treatments, with lower expression levels for G+NPS+EXO (F). CD31: Cluster of differentiation 31; EXO: Exosomes; G: Gel; NPS: Silver nanoparticles; SDA: Silver sulfadiazine; SMA: Smooth muscle actin. 


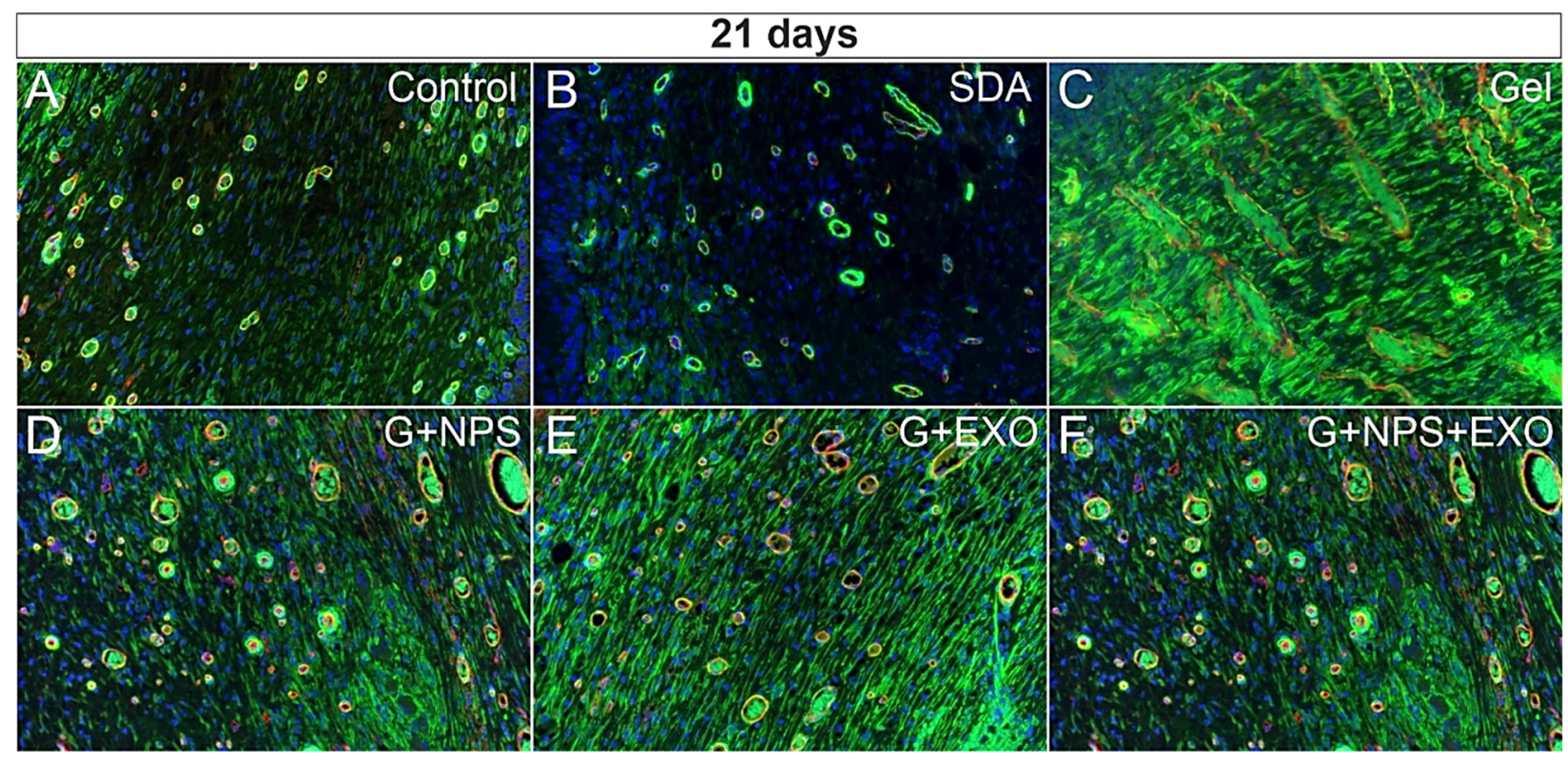

Figure $4-S M A$ (green) and CD31 (red) co-expression by fluorescence immunohistochemistry $(\times 200)$ in control group $(A)$ and the five treatment groups $(B-F)$ at 21 days after the lesion. An increase in the SMA immunoexpression was visible especially for simple gel (C) and G+EXO (E) groups. CD31: Cluster of differentiation 31; EXO: Exosomes; G: Gel; NPS: Silver nanoparticles; SDA: Silver sulfadiazine; SMA: Smooth muscle actin.
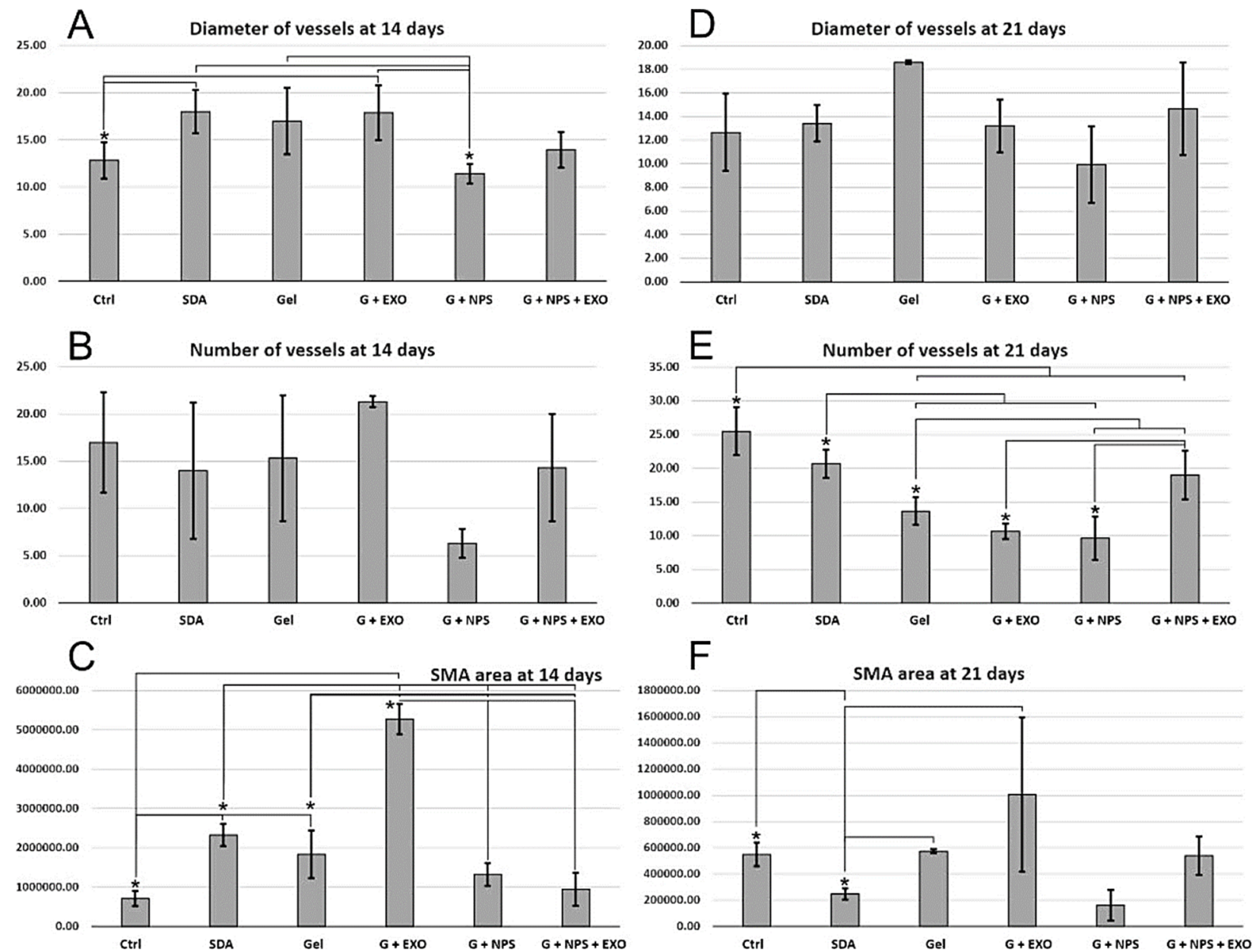

Figure 5 - Comparison between the five local treatments and the control (Ctrl) group. Vessel diameters (A and D), total number of vessels ( $B$ and $E$ ), as well as $S M A$ area $(C$ and $F$ ) have been evaluated in the granulation tissue beneath the epithelia at the lesion site. Vessel diameters show significant differences between groups at 14 days, the number of vessels were different at 21 days, and the SMA area recorded significant differences at both time points. Statistical differences were sought utilizing one-way ANOVA followed by Fisher's LSD test. *, $p<0.05$. Error bars represent standard deviation (SD) of the mean. ANOVA: Analysis of variance; EXO: Exosomes; G: Gel; LSD: Least significant difference; NPS: Silver nanoparticles; SDA: Silver sulfadiazine; SMA: Smooth muscle actin. 


\section{口 Discussions}

The skin has an area of about $1.8 \mathrm{~m}^{2}$, is the body's first line of defense against a multitude of external factors. The epidermis, consisting of a layered epithelium containing keratinocytes and melanocytes, provides a tight barrier to the external environment and prevents excessive water loss from the body. It also forms a barrier against ultraviolet (UV) radiation through the expression of melanin [15]. Keratinocytes express a number of lipids and antimicrobial peptides that control bacterial growth $[16,17]$.

Being a complex organ, exposed to external aggression factors, the skin has its own pathology from inflammatory processes to neoplastic processes, but its pathology can be the expression of systemic diseases (heart, kidney, respiratory, diabetes, etc.) [18-23].

Burns are among the most frequently occurring injuries $[3,24]$. The mechanisms of skin burns can be caused by a variety of factors, including heat (exposure to open flame, hot liquids, incandescent bodies), ionizing radiation, electricity, chemicals, etc. Burns cause the death of skin cells, both in the epidermis and in the dermis, leading to a massive loss of fluid from the body, followed by dehydration and electrolyte imbalance. Another serious complication is infection, because burned skin is extremely sensitive to bacteria and other pathogens, due to loss of protection by destroying the epidermis [3]. For these reasons, all studies show that it is essential to restore skin integrity as soon as possible.

Skin burns are a type of wound caused by thermal, electrical, chemical, or electromagnetic energy. Open flame is the most important cause of skin burns in the adults.

They are more common in men, as in some studies they accounted for up to three quarters of admitted cases [25] and the second phenomenon was a decrease in the incidence of large burns [26].

Managing a patient with severe burn injuries is a long-term process. The management addresses both local wound care and the immediate and progressive systemic response of injury [27]. Therefore, burns are among the most expensive traumatic injuries due to prolonged hospitalization, rehabilitation, physiotherapy costs and wound dressings. The costs of physiotherapy have been higher by longer duration and/or more frequent sessions physiotherapy to maintain joint movement and functionality [28]. Age is one of the strongest prognostic variables for mortality from burn injuries. The elderlies are more susceptible to burn injuries and the mortality rate is high even due to minor burns [28, 29].

Although accidents followed by skin burns can involve any region of the body, burns are most commonly seen in the upper and lower extremities [30, 31]. Patients with burns in the lower parts of the body are more likely to develop frequent complications, such as edema, deep vein thrombosis and pulmonary embolism, which increase hospitalization time and mortality rate [32].

There are many animal models utilized for studying skin burns, and the rodents are one of the most used model. Studies in mice showed that if the burns were dressed daily with either AgNPs or SDA over 28 days, the antibacterial effect of AgNPs and the speed of wound healing were better than those in the SDA group. A greater degree of inflammation was observed in groups of mice treated with SDA, with the presence of signs of inflammation, redness and swelling, which appeared to be reduced in wounds treated with AgNPs [33].

We have utilized here fibrin gel, polymeric AgNPs, and exosomes derived from human stromal mesenchymal cells, which have showed individual benefic previous effects in the wound healing process.

Histopathological analysis of the tissue repair process in mice that do not express fibrinogen, indicates significant microscopic changes, with persistent diffuse hemorrhage, and delayed re-epithelialization of wounds [34]. There is a deficiency in the formation of granulation tissue and the accumulated collagen fibers have low mechanical strength. Major changes occur in terms of cell repair dynamics, and in the case of skin lesions, keratinocytes do not systematically migrate from the periphery of the lesion to its center. The addition of a fibrinogen matrix in the middle of the lesion to these animals favors the migration of keratinocytes, emphasizing its pro-repair effect. Except the formation of fibrin, with an essential role in the process of hemostasis, fibrinogen also participates in other ways in the process of repairing lesions [35]. Fibrinogen undergoes conformational changes, exposing fibrin-like epitopes, and forms fibrillar elements of cellular anchoring in the structure of the temporary matrix that initially appears in the process of repairing skin and vascular lesions [36]. Thus, integrin molecules, cadherins or selectins, exposed on the membranes of endothelial cells, smooth muscle cells, keratinocytes, fibroblasts, or leukocytes, ensure this interaction [37, 38]. Also, fibrinogen can bind and maintain an increased level of growth factors (GFs), such as fibroblast growth factor-2 (FGF-2) and vascular endothelial growth factor (VEGF), favoring the appearance and maturation of granulation tissue [39]. Associated with the repair processes is the mixed inflammatory infiltrate (acute and chronic) that phagocytoses the necrotic elements and ensures tissue remodeling and defense against contaminating infectious agents. Fibrinogen and fibrin modulate the activity of monocytes and macrophages, favoring the maturation of granulation tissue with the decrease of inflammatory elements [40].

Exosomes derived from human stromal mesenchymal cells have been showed to express CD9, CD63 and CD81 on their surface.

Models of knock-out mice for CD9 show deficient reepithelialization of wounds, with persistent tissue dehiscence compared to batch of control animals [41]. It appears that the exact mechanism in these animals is prolonged activation of the c-Jun $\mathrm{NH}_{2}$-terminal kinase (JNK) in keratinocytes, with abnormally high levels of matrix metalloproteinase-9 (MMP-9), leading to excessive degradation of type IV collagen, and therefore to a defective formation of the vascular and epithelial basement membranes. CD63 is a receptor for the tissue inhibitor of metalloproteinase-1 (TIMP-1) and promotes cell survival, remodeling of the actin cytoskeleton, intercellular adhesion, and also the migration of keratinocytes, by activating protein kinase 2 
focal adhesion kinase (FAK). It accelerates angiogenesis processes by promoting the internalization of activated VEGF receptor 2 (VEGFR2) [42, 43].

CD81 promotes cell migration in a broad spectrum, both proteins being overexpressed in invasive and metastatic tumors [44]. Cell migration tests for wound healing have shown a localization of CD9 and CD81 at the intercellular junctions of mobile keratinocytes, but also an inhibition of cell migration following treatment with neutralizing antibodies to these molecules [45]. Although the exact mechanisms of how the two molecules are involved in wound repair are not known exactly, they appear to achieve transient adhesions and recycling of integrin receptors during keratinocyte migration.

Granulation tissue is a type of immature connective tissue that occurs during initial stages of wound repair, after hemostasis. It is characterized by proliferation of fibroblasts/myofibroblasts, angiogenesis, and the presence of inflammatory cells in a loose extracellular matrix. With time, granulation tissue matures up, the number of blood vessels decrease, and fibroblasts produce more and more collagen, till a scar is formed and the wound is re-epithelized on its surface.

Changes in the diameter of the blood vessels occur during wound repair and granulation tissue maturation and mediate the increase of blood flow to support hypoxic tissue that needs an increased blood supply to continue healing and surviving. These vessels need to provide more oxygen and nutrition supply to the damaged and hypoxic tissue, and their enlargement probable mediates that process and occurred due to the release of histamine by platelets [46].

Burn wound extract stimulate endothelial cells to produce inflammatory proteins and intensified endothelial cell migration. Moreover, burn wound extract have no effect on angiogenic sprouting and endothelial cell proliferation, and practically inhibited basic fibroblast growth factor (bFGF)mediated proliferation and sprouting. In contrast, burn wound extract stimulate both migration and proliferation of fibroblasts and adipose tissue-derived mesenchymal stromal cells [47].

With different stimuli $[$ e.g., transforming growth factorbeta (TGF- $\beta$ ) or mechanical tension], fibroblasts can express $\alpha$-SMA and form myofibroblasts with a contraction capacity. This enables wound contraction from day 6 post injury but is GF-dependent mechanism [48]. Myofibroblasts-derived $\alpha$-SMA has previously been correlated with acquisition of contractile phenotype and force generation [49]. Furthermore, $\alpha$-SMA was also observed to be directly correlated to scar thickness and re-epithelialization times. Higher levels of $\alpha$-SMA were found in thicker scars with delayed wound closure. On the $14^{\text {th }}$ day after burn, the level of $\alpha$-SMA expression in burn wounds was significantly related to the depth of burns and wound healing outcome. Statistical analysis suggested that the higher levels of $\alpha$-SMA in 6-week-old scars were found in smaller scars [50].

Although the study was limited to a relatively small number of animals due to the experimental complexity, our results are a promising premise for improved skin burn treatments aimed at both better repair and infection prevention.

\section{ㅁ Conclusions}

Our study is part of the efforts of researchers to develop strategies for effectively combating bacterial infection in skin burns, fluid loss, and wound healing. Our results showed that treatments with $\mathrm{G}+\mathrm{EXO}, \mathrm{G}+\mathrm{NPS}$ or $\mathrm{G}+\mathrm{NPS}+\mathrm{EXO}$, the burned wound recovered considerably faster compared to other topics applied as local treatments.

\section{Conflict of interests}

The authors declare that they have no conflict of interests.

\section{Acknowledgments}

This work was supported by a grant of the Romanian Ministry of Research and Innovation, CCDI - UEFISCDI, project number PN-III-P1-1.2-PCCDI-2017-0749/45PCCDI/ 2018, within PNCDI III ("Bioactive nanostructures for innovative therapeutic strategies").

\section{References}

[1] Nejati R, Kovacic D, Slominski A. Neuro-immune-endocrine functions of the skin: an overview. Expert Rev Dermatol, 2013, 8(6):581-583. https://doi.org/10.1586/17469872.2013.856690 PMID: 24587812 PMCID: PMC3938165

[2] Busuioc CJ, Mogoşanu GD, Popescu FC, Lascăr I, Pârvănescu H, Mogoantă $L$. Phases of the cutaneous angiogenesis process in experimental third-degree skin burns: histological and immunohistochemical study. Rom J Morphol Embryol, 2013, 54(1):163-171. PMID: 23529325

[3] Shpichka A, Butnaru D, Bezrukov EA, Sukhanov RB, Atala A, Burdukovskii $\vee$, Zhang Y, Timashev $P$. Skin tissue regeneration for burn injury. Stem Cell Res Ther, 2019, 10(1):94. https:// doi.org/10.1186/s13287-019-1203-3 PMID: 30876456 PMCID: PMC6419807

[4] Church D, Elsayed S, Reid O, Winston B, Lindsay R. Burn wound infections. Clin Microbiol Rev, 2006, 19(2):403-434. https://doi.org/10.1128/CMR.19.2.403-434.2006 PMID: 16614255 PMCID: PMC1471990

[5] Souto EB, Ribeiro AF, Ferreira MI, Teixeira MC, Shimojo AAM, Soriano JL, Naveros BC, Durazzo A, Lucarini M, Souto SB, Santini A. New nanotechnologies for the treatment and repair of skin burns infections. Int J Mol Sci, 2020, 21(2):393. https:// doi.org/10.3390/ijms21020393 PMID: 31936277 PMCID: PMC 7013843

[6] World Health Organization (WHO). Burns. WHO, Geneva, Switzerland, 2021. https://www.who.int/news-room/fact-sheets/ detail/burns

[7] Ansermino M, Hemsley C. Intensive care management and control of infection. BMJ, 2004, 329(7459):220-223. https:// doi.org/10.1136/bmj.329.7459.220 PMID: 15271835 PMCID: PMC487741

[8] Branski LK, Al-Mousawi A, Rivero H, Jeschke MG, Sanford AP, Herndon DN. Emerging infections in burns. Surg Infect (Larchmt), 2009, 10(5):389-397. https://doi.org/10.1089/sur.2009.024 PMID: 19810827 PMCID: PMC2956561

[9] Azzopardi EA, Azzopardi SM, Boyce DE, Dickson WA. Emerging Gram-negative infections in burn wounds. J Burn Care Res, 2011, 32(5):570-576. https://doi.org/10.1097/BCR.0b013e31 822ac7e6 PMID: 21792068

[10] Everett J, Turner K, Cai Q, Gordon V, Whiteley M, Rumbaugh K. Arginine is a critical substrate for the pathogenesis of Pseudomonas aeruginosa in burn wound infections. mBio, 2017, 8(2):e02160-16. https://doi.org/10.1128/mBio.02160-16 PMID: 28292986 PMCID: PMC5350470

[11] Santos JV, Viana J, Amarante J, Freitas A. Paediatric burn unit in Portugal: beds needed using a bed-day approach. Burns, 2017, 43(2):403-410. https://doi.org/10.1016/j.burns.2016.08. 014 PMID: 27644139

[12] Rafla K, Tredget EE. Infection control in the burn unit. Burns, 2011, 7(1):5-15. https://doi.org/10.1016/j.burns.2009.06.198 PMID: 20561750 
[13] Tiwari VK. Burn wound: how it differs from other wounds? Indian J Plast Surg, 2012, 45(2):364-373. https://doi.org/10. 4103/0970-0358.101319 PMID: 23162236 PMCID: PMC 3495387

[14] Mofazzal Jahromi MA, Sahandi Zangabad P, Moosavi Basri SM, Sahandi Zangabad K, Ghamarypour A, Aref AR, Karimi M, Hamblin MR. Nanomedicine and advanced technologies for burns: preventing infection and facilitating wound healing. Adv Drug Deliv Rev, 2018, 123:33-64. https://doi.org/10.1016/j. addr.2017.08.001 PMID: 28782570 PMCID: PMC5742034

[15] Chambers ES, Vukmanovic-Stejic M. Skin barrier immunity and ageing. Immunology, 2020, 160(2):116-125. https://doi.org/ 10.1111/imm.13152 PMID: 31709535 PMCID: PMC7218662

[16] Fulton C, Anderson GM, Zasloff M, Bull R, Quinn AG. Expression of natural peptide antibiotics in human skin. Lancet, 1997 350(9093):1750-1751. https://doi.org/10.1016/S0140-6736(05) 63574-X PMID: 9413472

[17] Wertz PW. Lipids and the permeability and antimicrobial barriers of the skin. J Lipids, 2018, 2018:5954034. https://doi.org/10. 1155/2018/5954034 PMID: 30245886 PMCID: PMC6139190

[18] Gallagher RP, Lee TK. Adverse effects of ultraviolet radiation: a brief review. Prog Biophys Mol Biol, 2006, 92(1):119-131. https://doi.org/10.1016/j.pbiomolbio.2006.02.011 PMID: 16580054

[19] Rotaru M, lancu GM, Gheucă Solovăstru L, Glaja RF, Grosu F, Bold A, Costache A. A rare case of multiple clear cell acanthoma with a relatively rapid development of the lower legs. Rom J Morphol Embryol, 2014, 55(3 Suppl):1171-1179. PMID: 25607402

[20] Guha N, Loomis D, Guyton KZ, Grosse Y, El Ghissassi F, Bouvard V, Benbrahim-Tallaa L, Vilahur N, Muller K, Straif K; International Agency for Research on Cancer Monograph Working Group. Carcinogenicity of welding, molybdenum trioxide, and indium tin oxide. Lancet Oncol, 2017, 18(5):581-582. https:// doi.org/10.1016/S1470-2045(17)30255-3 PMID: 28408286

[21] Lora V, Cerroni L, Cota C. Skin manifestations of rheumatoid arthritis. G Ital Dermatol Venereol, 2018, 153(2):243-255. https:// doi.org/10.23736/S0392-0488.18.05872-8 PMID: 29368864

[22] Rendon A, Schäkel K. Psoriasis pathogenesis and treatment. Int J Mol Sci, 2019, 20(6):1475. https://doi.org/10.3390/ijms 20061475 PMID: 30909615 PMCID: PMC6471628

[23] Abenavoli L, Dastoli S, Bennardo L, Boccuto L, Passante M, Silvestri M, Proietti I, Potenza C, Luzza F, Nisticò SP. The skin in celiac disease patients: the other side of the coin. Medicina (Kaunas), 2019, 55(9):578. https://doi.org/10.3390/ medicina55090578 PMID: 31505858 PMCID: PMC6780714

[24] Hettiaratchy S, Dziewulski P. ABC of burns: pathophysiology and types of burns. BMJ, 2004, 328(7453):1427-1429. https:// doi.org/10.1136/bmj.328.7453.1427. Erratum in: BMJ, 2004, 329(7458):148. PMID: 15191982 PMCID: PMC421790

[25] Nickel KJ, Omeis T, Papp A. Demographics and clinical outcomes of adult burn patients admitted to a single provincial burn centre: a 40-year review. Burns, 2020, 46(8):1958-1967. https://doi.org/10.1016/j.burns.2020.06.020 PMID: 32660831

[26] Anwar U, Majumder S, Austin O, Phipps AR. Changing pattern of adult burn referrals to a regional burns centre. J Burn Care Res, 2007, 28(2):299-305. https://doi.org/10.1097/BCR.0B0 13E318031A106 PMID: 17351448

[27] Vigani A, Culler CA. Systemic and local management of burn wounds. Vet Clin North Am Small Anim Pract, 2017, 47(6): 1149-1163. https://doi.org/10.1016/j.cvsm.2017.06.003 PMID: 28802983

[28] Patil V, Dulhunty JM, Udy A, Thomas P, Kucharski G, Lipman J. Do burn patients cost more? The intensive care unit costs of burn patients compared with controls matched for length of stay and acuity. J Burn Care Res, 2010, 31(4):598-602. https:// doi.org/10.1097/BCR.0b013e3181e4d6a4 PMID: 20489652

[29] Sarhadi NS, Kincaid R, McGregor JC, Watson JD. Burns in the elderly in the south east of Scotland: review of 176 patients treated in the Bangour Burns Unit (1982-91) and burn inpatients in the region (1975-91). Burns, 1995, 21(2):91-95. https://doi.org/ 10.1016/0305-4179(95)92130-5 PMID: 7766332

[30] Pham TN, Cancio LC, Gibran NS; American Burn Association. American Burn Association practice guidelines burn shock resuscitation. J Burn Care Res, 2008, 29(1):257-266. https:// doi.org/10.1097/BCR.0b013e31815f3876 PMID: 18182930

[31] Brusselaers N, Monstrey S, Vogelaers D, Hoste E, Blot S. Severe burn injury in Europe: a systematic review of the incidence, etiology, morbidity, and mortality. Crit Care, 2010,
14(5):R188. https://doi.org/10.1186/cc9300 PMID: 20958968 PMCID: PMC3219295

[32] Mohammadi AA, Pakyari MR, Seyed Jafari SM, Tavakkolian AR, Tolide-le HR, Moradi Z, Kherad M. Effect of burn sites (upper and lower body parts) and gender on extensive burns' mortality. Iran J Med Sci, 2015, 40(2):166-169. PMID: 25821297 PMCID: PMC4359937

[33] Wasef LG, Shaheen HM, El-Sayed YS, Shalaby TIA, Samak DH, Abd El-Hack ME, Al-Owaimer A, Saadeldin IM, El-Mleeh A, Ba-Awadh $\mathrm{H}$, Swelum AA. Effects of silver nanoparticles on burn wound healing in a mouse model. Biol Trace Elem Res, 2020, 193(2):456-465. https://doi.org/10.1007/s12011-01901729-z PMID: 31111309

[34] Drew AF, Liu H, Davidson JM, Daugherty CC, Degen JL. Wound-healing defects in mice lacking fibrinogen. Blood, 2001, 97(12):3691-3698. https://doi.org/10.1182/blood.v97.12.3691 PMID: 11389004

[35] Laurens N, Koolwijk P, de Maat MPM. Fibrin structure and wound healing. J Thromb Haemost, 2006, 4(5):932-939. https:// doi.org/10.1111/j.1538-7836.2006.01861.x PMID: 16689737

[36] Guadiz G, Sporn LA, Simpson-Haidaris PJ. Thrombin cleavageindependent deposition of fibrinogen in extracellular matrices. Blood, 1997, 90(7):2644-2653. https://doi.org/10.1182/blood. V90.7.2644 PMID: 9326231

[37] Bennett JS. Platelet-fibrinogen interactions. Ann N Y Acad Sci, 2001, 936:340-354. https://doi.org/10.1111/j.1749-6632.2001. tb03521.x PMID: 11460491

[38] Martinez J, Ferber A, Bach TL, Yaen CH. Interaction of fibrin with VE-cadherin. Ann N Y Acad Sci, 2001, 936:386-405. https://doi.org/10.1111/j.1749-6632.2001.tb03524.x PMID: 11460494

[39] Rybarczyk BJ, Lawrence SO, Simpson-Haidaris PJ. Matrixfibrinogen enhances wound closure by increasing both cell proliferation and migration. Blood, 2003, 102(12):4035-4043. https://doi.org/10.1182/blood-2003-03-0822 PMID: 12920033

[40] Kubo M, Van de Water L, Plantefaber LC, Mosesson MW, Simon M, Tonnesen MG, Taichman L, Clark RA. Fibrinogen and fibrin are anti-adhesive for keratinocytes: a mechanism for fibrin eschar slough during wound repair. J Invest Dermatol, 2001, 117(6):1369-1381. https://doi.org/10.1046/j.0022-202x. 2001.01551.x PMID: 11886497

[41] Zhang J, Dong J, Gu H, Yu S, Zhang X, Gou Y, Xu W, Burd A, Huang L, Miyado K, Huang $Y$, Chan HC. CD9 is critical for cutaneous wound healing through JNK signaling. J Invest Dermatol, 2012, 132(1):226-236. https://doi.org/10.1038/jid. 2011.268 PMID: 21881583

[42] Jung KK, Liu XW, Chirco R, Fridman R, Kim HRC. Identification of CD63 as a tissue inhibitor of metalloproteinase-1 interacting cell surface protein. EMBO J, 2006, 25(17):3934-3942. https:// doi.org/10.1038/sj.emboj.7601281 PMID: 16917503 PMCID: PMC1560352

[43] Lee SY, Kim JM, Cho SY, Kim HS, Shin HS, Jeon JY, Kausar R, Jeong SY, Lee YS, Lee MA. TIMP-1 modulates chemotaxis of human neural stem cells through CD63 and integrin signalling. Biochem J, 2014, 459(3):565-576. https://doi.org/10.1042/ BJ20131119 PMID: 24635319

[44] Mizoshiri N, Shirai T, Terauchi R, Tsuchida S, Mori Y, Hayashi D, Kishida T, Arai Y, Mazda O, Nakanishi T, Kubo T. The tetraspanin CD81 mediates the growth and metastases of human osteosarcoma. Cell Oncol (Dordr), 2019, 42(6): 861-871. https://doi.org/10.1007/s13402-019-00472-w PMID: 31494861

[45] Peñas PF, García-Díez A, Sánchez-Madrid F, Yáñez-Mó M. Tetraspanins are localized at motility-related structures and involved in normal human keratinocyte wound healing migration. J Invest Dermatol, 2000, 114(6):1126-1135. https://doi.org/ 10.1046/j.1523-1747.2000.00998.x PMID: 10844555

[46] Yousefi S, Qin J, Dziennis S, Wang RK. Assessment of microcirculation dynamics during cutaneous wound healing phases in vivo using optical microangiography. J Biomed Opt, 2014, 19(7):76015. https://doi.org/10.1117/1.JBO.19.7.0760 15 PMID: 25036212 PMCID: PMC4103582

[47] Monsuur HN, van den Broek LJ, Jhingoerie RL, Vloemans AFPM, Gibbs S. Burn eschar stimulates fibroblast and adipose mesenchymal stromal cell proliferation and migration but inhibits endothelial cell sprouting. Int J Mol Sci, 2017, 18(8):1790. https://doi.org/10.3390/ijms18081790 PMID: 28820426 PMCID: PMC5578178 
[48] Greaves NS, Ashcroft KJ, Baguneid M, Bayat A. Current understanding of molecular and cellular mechanisms in fibroplasia and angiogenesis during acute wound healing. J Dermatol Sci, 2013, 72(3):206-217. https://doi.org/10.1016/ j.jdermsci.2013.07.008 PMID: 23958517

[49] Hinz B, Celetta G, Tomasek JJ, Gabbiani G, Chaponnier C. Alpha-smooth muscle actin expression upregulates fibroblast contractile activity. Mol Biol Cell, 2001, 12(9):2730-2741. https:// doi.org/10.1091/mbc.12.9.2730 PMID: 11553712 PMCID: PMC59708

[50] Wang XQ, Kravchuk O, Winterford C, Kimble RM. The correlation of in vivo burn scar contraction with the level of $\alpha$-smooth muscle actin expression. Burns, 2011, 37(8):13671377. https://doi.org/10.1016/j.burns.2011.07.018 PMID: 21855218

\section{Corresponding author}

Elena Leocadia Popescu, MD, PhD Student, Doctoral School, University of Medicine and Pharmacy of Craiova, 2 Petru Rareş Street, 200349 Craiova, Dolj County, Romania; Phone +40767-815 259, e-mail: popescu.elena88@yahoo.com

Received: March 30, 2021

Accepted: September 27, 2021 